\title{
PBTA - Projeto Baseado em Trajetórias de Aprendizagem
}

\author{
Alberto Bastos do Canto Filho - alberto.canto@ufrgs.br - PPGIE - UFRGS \\ José Valdeni de Lima - valdeni@inf.ufrgs.br - PPGIE - UFRGS \\ Eráclito de Souza Argolo - esargolo@gmail.com - PPGIE - UFRGS \\ Liane Margarida Rockenbach Tarouco - liane@penta.ufrgs.br - PPGIE - UFRGS
}

Resumo: A contribuição deste artigo é a apresentação de um método para o projeto de Objeto de Aprendizagem baseado em trajetórias de aprendizagem. Este método foi criado a partir de três princípios de projeto de objetos de aprendizagem: Princípio dos Subsunçores, Princípio da Granularidade e Princípio do Ajuste de Ritmo. A aplicação destes três princípios resulta em dois tipos de segmentação: segmentação interobjetos, e segmentação intraobjetos. A segmentação interobjeto define a arquitetura do sistema educacional, em termos de objetos de aprendizagem projetados; A segmentação intraobjeto define o sistema de navegação de um dentro de um mesmo objeto. As alternativas de trajetória de aprendizagem do sistema educacional são resultantes deste processo de segmentação.

Palavras Chave - Objetos de Aprendizagem, projeto, Trajetória de Aprendizagem

Abstract: The contribution of this paper is a methodology called 'Project Based on Learning Trajectories'. This method is based on three project principles: Subsumers Principle, Granularity Principle and Rhythm Adjust Principle. The use of this principles results in two kinds of segmentation: interobjects and intraobject. The interobject segmentation defines the system architecture in terms of learning objects. The intraobject segmentation defines the navigational system of a specific learning object. The results of this segmentation process are the possible learning trajectories.

Keywords - Learning Object, Project Principles, Methodology

\section{Introdução}

Uma das condições essências para o aprendizado significativo é a utilização de materiais potencialmente significativos (AUSUBEL, NOVAK e HANESIAN, 1978). A aplicação prática desta premissa relativamente a Objetos de Aprendizagem (OA) encontra obstáculos no fato de que, devido a características individuais distintas, um determinado OA poderá ser potencialmente significativo apenas para uma parcela dos estudantes de uma turma. A condição ideal é aquela em que cada estudante segue uma trajetória de aprendizagem própria, como ocorre na tutoria individual, onde é possível obter resultados até dois sigmas superiores àqueles obtidos em turmas com trinta estudantes (BLOOM, 1984).

Segundo Ausubel et al (1978) o aspecto de maior relevância para aprendizado significativo é a existência de conceitos previamente conhecidos (conceitos subsunçores) com os quais as novas proposições sejam relacionadas de forma substantiva e não arbitrária. Esta necessária existência de conceitos subsunçores como um ponto de partida, associada à premissa de existência de um objetivo educacional como ponto de chegada permite que se compare o projeto de objetos de aprendizagem com o projeto de uma malha de acessos entre conceitos. Esta analogia é especialmente válida na educação na educação superior, onde adultos que já possuam uma base 
conceitual formada aprendem por processos de diferenciação ou integração aos conceitos prévios.

Neste artigo são apresentadas formas práticas de definição de trajetórias de aprendizagem a partir de três princípios de projeto de objetos: Princípio dos Subsunçores, Princípio da Granularidade e Princípio do Ajuste de Ritmo.

\section{Trabalhos Prévios}

O presente trabalho insere-se no contexto de investigação sobre princípios de projeto de objetos de aprendizagem, que tem como principal motivação o uso de tecnologia na educação com vistas à redução do percentual de estudantes de engenharia que abandona o curso; este percentual é da ordem de $40 \%$ no curso investigado.

A partir dos princípios de projeto existentes na literatura (MAYER, 2005) (MORENO e MAYER, 2007) (VAN MERRIËNBOER e SWELLER, 2010), e de princípios de projeto propostos foi desenvolvido um objeto de aprendizagem utilizado numa investigação que comparou os resultados obtidos por estudantes que aprenderam no sistema tradicional (aulas expositivas) com os resultados dos estudantes que aprenderam utilizando o Objeto de Aprendizagem (CANTO et al., 2012) (CANTO, et al, 2013a) (CANTO et al, 2013b). Os resultados encontrados nesta investigação mostram que OAs adequadamente projetados e utilizados podem ser mais efetivos do que as aulas tradicionais.

Um dos motivos para este potencial ganho de eficácia é a possibilidade de introdução em larga escala de alguns elementos da tutoria individual, entre eles a possibilidade de ajuste individual de trajetória.

A seguir serão apresentados três princípios de projeto que vem sendo utilizados de forma integrada para o ajuste de trajetórias.

\section{Integração de Princípios de Projeto}

\subsection{Princípio dos Subsunçores}

O principal método de ensino e aprendizagem utilizado no âmbito do curso de engenharia investigado é a aprendizagem por recepção, o que remete à teoria da aprendizagem significativa (AUSUBEL, NOVAK e HANESIAN, 1978). Os principais motivos para a adoção desta alternativa são os seguintes:

Motivos econômicos: a aprendizagem por recepção permite uma elevada relação alunos/professor;

Motivos curriculares: com uma alta densidade de conteúdos semanalmente previstos, muitos professores temem não vencer o conteúdo programático caso venham a adotar alternativas de aprendizagem por descoberta (FELDER e PRINCE, 2006);

Premissa de maturidade cognitiva: Ausubel sustenta que a aprendizagem significativa por recepção é o método mais eficiente de aquisição de conhecimento, que emerge nos estágios mais avançados de maturidade cognitiva; ao assumir que um curso de engenharia é um curso de nível superior, muitos docentes assumem também que todos os estudantes estão (ou deveriam estar) suficientemente maduros para o aprendizado por recepção;

Aderência da Teoria: Ausubel (1978) define Aprendizado Significativo como o processo através do qual uma nova informação (um novo conhecimento) se relaciona de maneira não arbitrária e substantiva à estrutura cognitiva do aprendiz. Com uma estrutura conceitual altamente hierarquizada, nos cursos de 
engenharia o aprendizado de um conceito depende dos conhecimentos prévios, exigindo-se desde o início do curso uma sólida base de conhecimentos de matemática e física acumulados ao longo de toda a vida estudantil.

A eficácia e eficiência ${ }^{1}$ dos métodos de aprendizagem por recepção é uma realidade para uma parte dos estudantes que se adaptam perfeitamente a este método. No entanto, uma parcela significativa dos ingressantes (cerca de 40\%) não se ajusta aos cursos de engenharia investigados e terminam por evadir, especialmente nas etapas iniciais.

A quantidade e qualidade dos subsunçores ${ }^{2}$ são consideradas por Ausubel como o fator de maior relevância na aprendizagem significativa, razão pela qual é proposto o Princípio dos Subsunçores (CANTO et al., 2012) apresentado no Quadro 3-1.

Objetos de Aprendizagem são mais eficazes quando aplicados a estudantes que possuam subsunçores em quantidade e qualidade adequados aos objetivos educacionais estabelecidos.

Quadro 3-1. Princípio dos Subsunçores

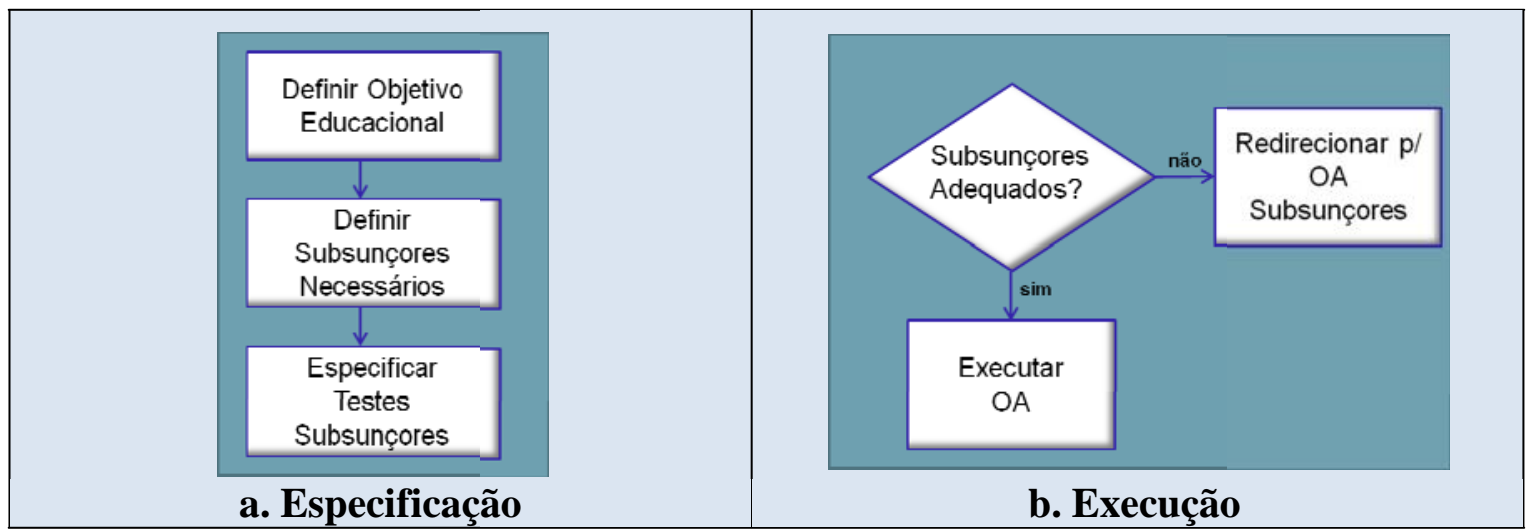

Figura 3.1 Fluxogramas de especificação e execução de OAs

A aplicação do princípio dos subsunçores no projeto de um objeto de aprendizagem é feita através da delimitação das condições de contorno da trajetória de aprendizagem, com a especificação clara dos objetivos educacionais (ponto de chegada) e dos conceitos subsunçores necessários (ponto de partida).

A delimitação do ponto inicial da trajetória é, portanto condição necessária por ocasião do projeto de um OA, sendo possível prever que o próprio OA efetue testes verificando se esta condição é atendida pelo estudante, conforme apresenta a Figura 3.1 (CANTO et al, 2013).

${ }^{1}$ Eficácia é definida como a extensão na qual as atividades planejadas são realizadas e os resultados planejados alcançados.

Eficiência é a relação entre o resultado alcançado e os recursos utilizados (ABNT ASSOCIAÇÃO BRASILEIRA DE NORMAS TECNICAS, 2005)

2 Conceitos Subsunçores: conceito, ideia ou proposição previamente existente na estrutura cognitiva como os quais as novas informações devem-se relacionar (AUSUBEL et al, 1978) 
Alternativamente, a informação sobre os conceitos subsunçores requeridos pode ser anexada ao OA na forma de documentação ou metadados. A Figura 3.2 mostra a tela de cadastramento de um novo Projeto de Objeto de Aprendizagem no sistema $A B C$ Autoria, um sistema CAD para objetos de aprendizagem que vem sendo desenvolvido no contexto desta investigação. Observe a incorporação do princípio dos subsunçores como um requisito de projeto através do qual se delimitam os pontos de partida ${ }^{3}$ e de chegada da trajetória de aprendizagem.

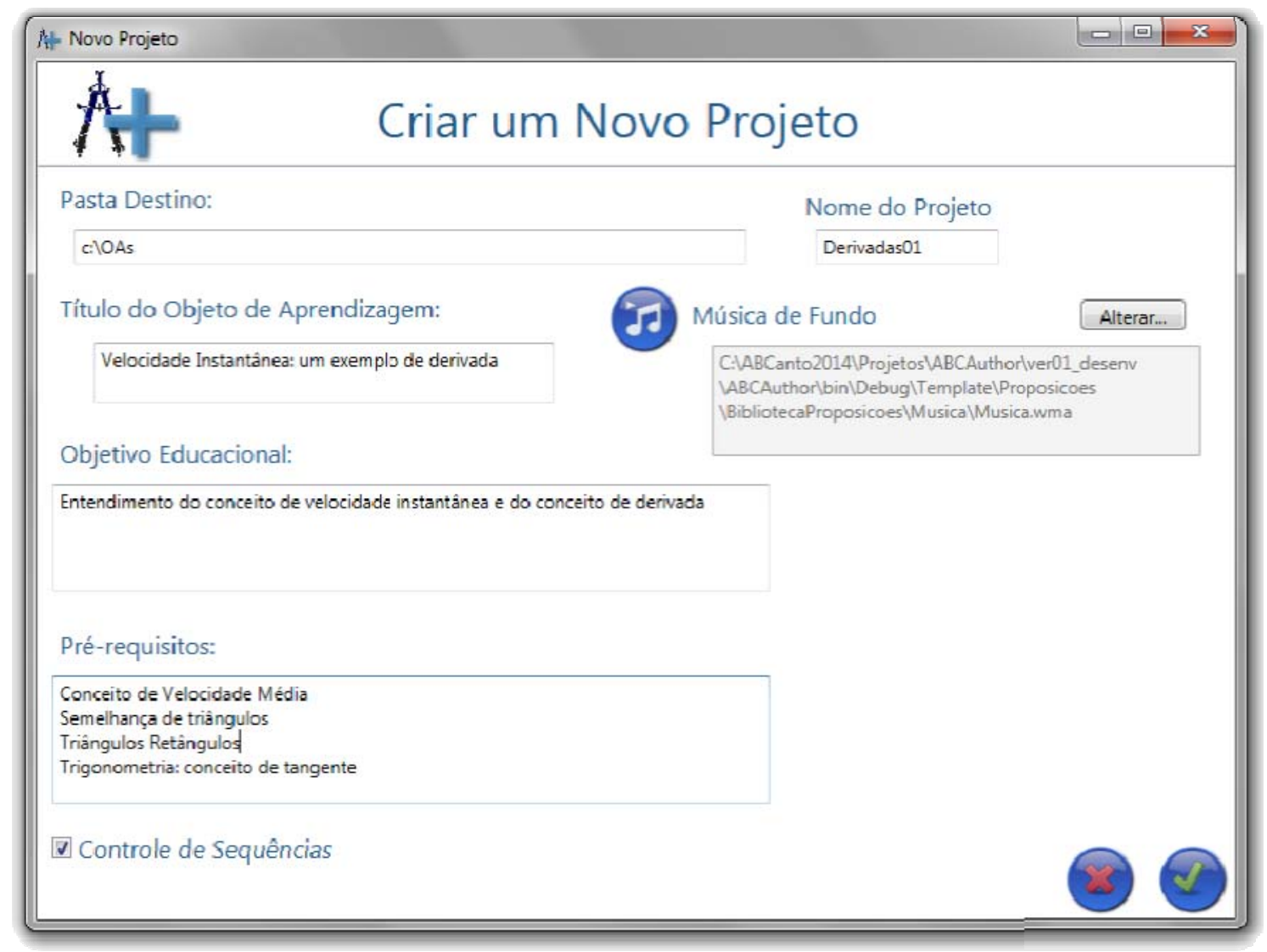

Figura 3.2 ABC Autoria - Cadastramento de um novo projeto

A aplicação do princípio dos subsunçores parte de um conceito relacionado à Teoria do Aprendizado Significativo, e define o escopo delimitador da trajetória de aprendizagem cujo objetivo é conectar conceitos. Esta clara delimitação de pontos de partida e pontos de chegada especificados para a definição da trajetória de um objeto de aprendizagem poderá ser planejada na forma de um sistema de aprendizagem, onde os conceitos apreendidos em um objeto de aprendizagem sejam conceitos subsunçores de outros objetos, criando assim uma malha de trajetórias de aprendizagem. Desta forma, o planejamento de um curso ou disciplina poderá ser realizado através de uma segmentação interobjetos.

\footnotetext{
3 Em sua primeira implementação esta tela utilizava o rótulo “Conceitos Subsunçores”; No entanto, optou-se por substituí-la por "Pré-requisitos” por ser uma expressão mais conhecida que, mesmo não sendo sinônimo, expressa a ideia de "ponto de partida" da trajetória de aprendizagem que será projetada no OA.
} 


\subsection{Princípio da Granularidade}

Embora possa apresentar resultados semelhantes àqueles decorrentes da aplicação do Princípio dos Subsunçores, a segmentação com base no Princípio da Granularidade (Quadro 3-2) é realizada por motivos diferentes.

Objetos de Aprendizagem segmentados em objetivos parciais proporcionam maior velocidade de desenvolvimento, maiores possibilidades de reutilização, e reduzem os riscos de sobrecarga cognitiva.

\section{Quadro 3-2. Princípio da Granularidade (CANTO et al, 2013)}

Existem dois motivos principais para a aplicação do Princípio da Granularidade:

Racionalização do projeto: a divisão de um projeto complexo em subprojetos menores e mais simples permite que diversas equipes trabalhem em paralelo, reduzindo assim os prazos de entrega. A menor complexidade dos módulos representam menores riscos, admitindo que se trabalhe com profissionais menos experientes. Paralelamente, maior granularidade aumenta a possibilidade de reutilização com consequente redução de custos de desenvolvimento (TAROUCO et al, 2011);

Redução do Risco de Sobrecarga Cognitiva: a sobrecarga cognitiva ocorre quando há uma demanda de recursos cognitivos superior aos recursos disponíveis (SWELLER, 1988), e pode ser explicada com base na Figura 3.3.

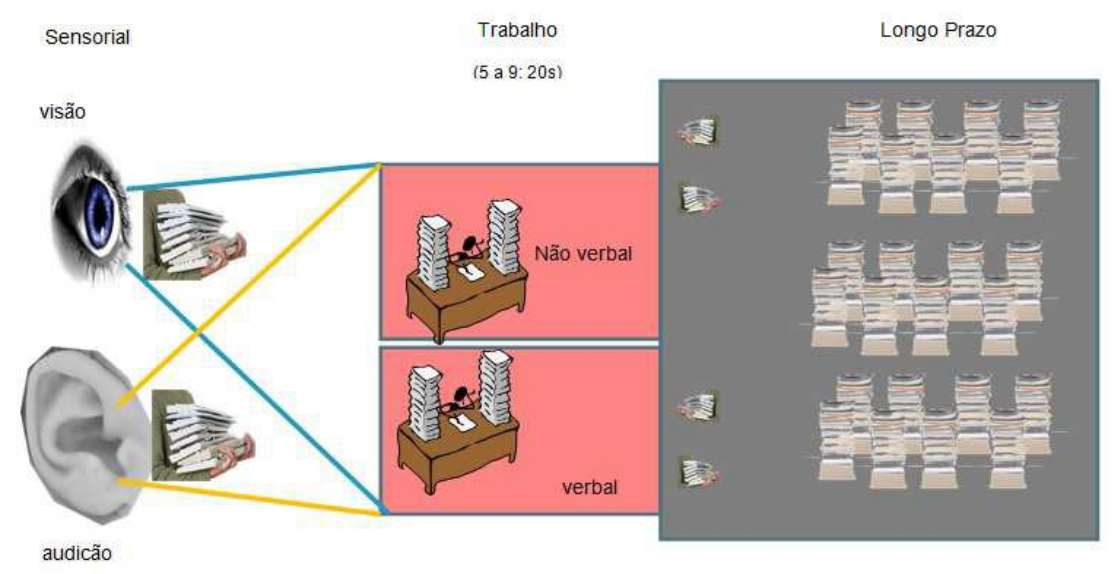

Figura 3.3. Arquitetura Cognitiva Humana (baseado em (MAYER, 2005))

A Figura 3.3 apresenta a arquitetura cognitiva humana subdividida em três tipos de memória: Memória Sensorial, Memória de Trabalho e Memória de Longo prazo. Todo o processamento é realizado na memória de trabalho, que possui limitações quanto ao número de elementos que podem ser tratadas simultaneamente. A memória de trabalho pode lidar com número máximo de cinco a nove elementos simultâneos, mas este número se reduz para um valor entre dois e quatro quando existe a necessidade de algum tipo de processamento tal como organizar, comparar, ou qualquer outro tratamento (VAN MERRIËNBOER e SWELLER, 2010) 
O aprendizado significativo é necessariamente um aprendizado ativo, pois requer diversas operações cognitivas referentes à análise crítica das novas proposições ${ }^{4}$ frente aos conceitos subsunçores. A consequência deste aprendizado ativo é a maior carga da memória de trabalho, impondo uma redução do número de elementos de informação tratados simultaneamente, o que pode ser feito através de uma segmentação que distribua as proposições ao longo do tempo.

Esta segmentação de proposições ao longo do tempo tem um caráter de encadeamento de ideias, e é realizada dentro de um mesmo objeto de aprendizagem: segmentação intraobjeto.

\subsection{Princípio do Ajuste de Ritmo}

Além das limitações relacionadas ao número de elementos simultâneos, a memória de trabalho possui também limitações temporais: as novas proposições, recebidas através dos sentidos (memória sensorial) são perdidas cerca de 20 segundos após o último acesso (VAN MERRIËNBOER e SWELLER, 2010). O Princípio do Ajuste de Ritmo apresentado no Quadro 3-3 contempla a necessidade de que a segmentação de conteúdos não se preocupe apenas com o número de elementos tratados simultaneamente, mas também com a necessidade de tempo para o processamento das novas informações, ancorando-as aos conceitos subsunçores enquanto ainda disponíveis na memória de trabalho.

Objetos de Aprendizagem são mais eficazes quando permitem que o estudante ajuste o ritmo de execução ao seu ritmo de aprendizagem.

\section{Quadro 3-3. Princípio do Ajuste de Ritmo (CANTO et al, 2013)}

No contexto do sistema $A B C$ Autoria foram especificados padrões de projeto que contemplam diversos princípios de projeto. O padrão denominado 'Proposição Sequência' permite a implementação de proposições complexas respeitando as limitações da memória de trabalho, tanto no que diz respeito ao número de elementos simultâneos quanto no que se refere a limitações temporais.

A "Proposição Sequência" é definida de forma recursiva: Proposição sequência é uma sequência de proposições. O código que o sistema $A B C$ Autoria gera para as proposições sequência prevê que a sequência de execução seja interrompida após a execução de cada segmento, exigindo uma solicitação de 'próxima proposição', para que o próximo segmento seja executado. A Proposição Sequência permite que cada estudante tome o tempo que necessitar para realizar as operações cognitivas necessárias para o aprendizado significativo das proposições trabalhadas antes que novas proposições demandem a sua memória de trabalho. Cabe ao designer instrucional projetar cada trecho da sequência de forma a não sobrecarregar a memória de trabalho. Recomenda-se que as exposições de ideias sejam segmentadas em trechos com duração da ordem de 20 segundos, limitando assim o número máximo de proposições simultâneas e reduzindo a possibilidade de as novas proposições sejam perdidas devido às limitações temporais da memória de trabalho.

4 Ausubel (1978) define 'Proposição' como uma descrição da realidade criada pelo homem. No contexto da investigação realizada, foi utilizada a seguinte definição: Descrição da realidade apresentada pelo Objeto de Aprendizagem. Esta definição inclui não apenas a aprendizagem receptiva de uma descrição da realidade, mas também a possibilidade de que um Objeto de Aprendizagem descreva a realidade através de atividades interativas que proporcionem o aprendizado por descoberta. 


\section{Trajetórias Interobjetos e Intraobjeto}

O projeto de Objetos de Aprendizagem baseado em trajetórias planejadas possui dois níveis de segmentação:

Interobjetos: quando um espaço pedagógico ${ }^{5}$ é segmentado em diversos Objetos de Aprendizagem.

Intraobjeto: quando um Objeto de Aprendizagem é segmentado em diversas proposições.

Cabe ao designer instrucional definir os diversos objetivos educacionais (Conceitos) de seu espaço de aprendizagem e a forma como estes se conectam.

A Figura 4.1 apresenta um exemplo de um espaço pedagógico que vem sendo criado com o objetivo de disseminar os resultados da pesquisa na qual se insere este trabalho. O exemplo mostra diversos conceitos conectados entre si através de setas que representam proposições, definindo as rotas de aprendizagem da proposição chamada Introdução. Esta proposição é uma composição de três proposições, que podem ser acessadas através de um menu: Questão de Pesquisa; Eficácia X Eficiência; Resumo. A Proposição 'Questão de Pesquisa' é uma sequência de proposições multimídia multimodais.

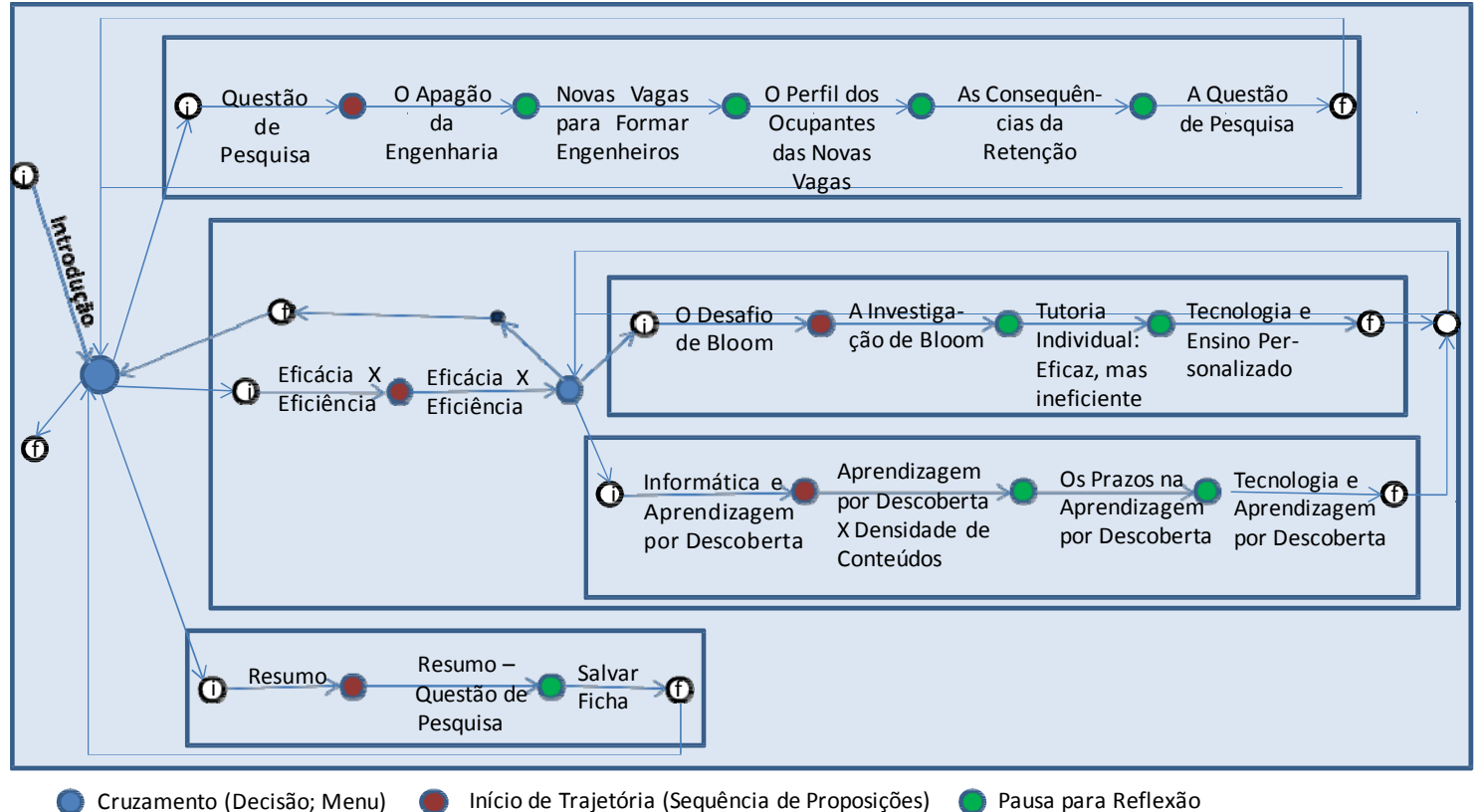

Figura 4.1. Exemplo de Espaço Pedagógico

Cada uma das Proposições Mãe $e^{6}$ está delimitada por um retângulo azul, com conectores brancos que demarcam o início e o fim da proposição. Esta delimitação tem por objetivo estabelecer pontos de conexão para reuso futuro. Por exemplo, a motivação

${ }^{5}$ Define-se aqui “Espaço Pedagógico” como uma unidade de Ensino e Aprendizagem que utiliza tecnologia da informação para abordar diversos conceitos inter-relacionados. O número de conceitos contidos definirá o tamanho do Espaço Pedagógico, que poderá englobar desde um único conceito até um curso completo.

${ }^{6}$ Proposição Mãe é uma proposição composta por outras proposições. Na versão atual do sistema $A B C$ Autoria estão previstas duas classes de Proposição Mãe: Proposição Menu (que implementa os cruzamentos na trajetória) e a Proposição Sequência (que implementa uma sequência de Proposições). 
baseada nas pesquisas de Bloom (1987), será reutilizada por outros OAs que queiram explorar esta mesma motivação.

\section{Conclusão}

O método de Projeto de Objetos de Aprendizagem baseado e trajetórias baseiase em princípios de projeto princípios de projeto existentes na literatura (MAYER, 2005) (MORENO e MAYER, 2007) (VAN MERRIËNBOER e SWELLER, 2010), bem como princípios de projeto propostos no contexto desta pesquisa. Esta metodologia vem sendo implementada no sistema $A B C$ Autoria, um CAD para o projeto de Objetos de Aprendizagem. A Figura 5.1 mostra a tela do sistema $A B C$ Autoria utilizada para a inserção do modelo apresentado na Figura 4.1. O objeto produzido pode ser consultado em http://chasqueweb.ufrgs.br/ alberto.canto/TeseDr/Motivacao/.

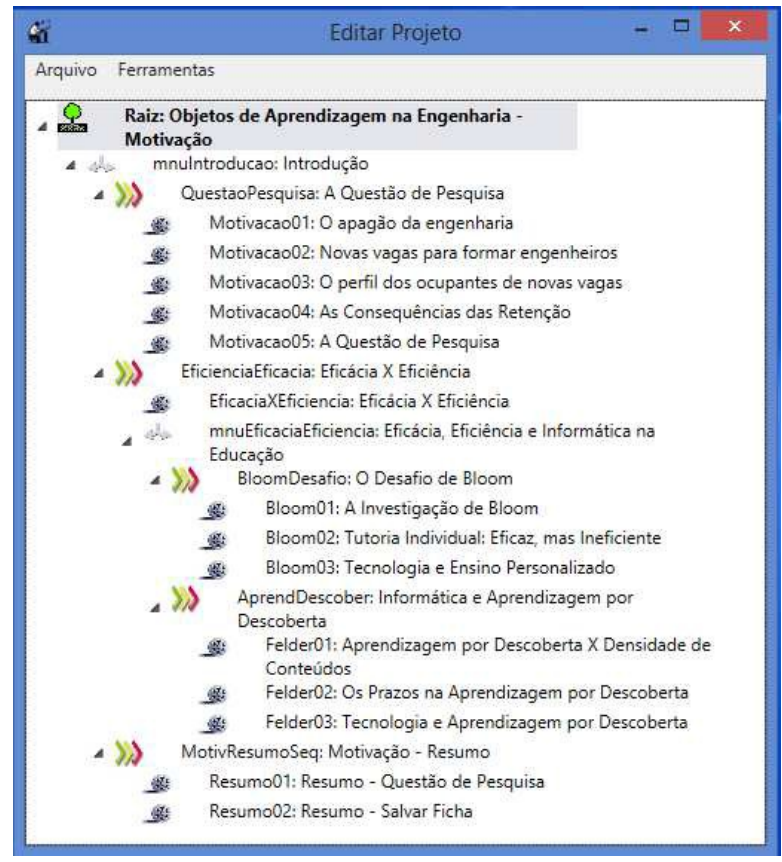

\section{Figura 5.1. ABC Autoria - Projeto Baseado em Trajetórias}

A criação de métodos e ferramentas que simplifiquem o projeto e implementação de Objetos de Aprendizagem é condição essencial para a viabilização econômica do uso de Tecnologia na Educação.

A metodologia proposta associada a uma ferramenta CAD permite que se criem objetos de aprendizagem sem a necessidade de esforço de programação, permitindo assim que se concentrem esforços na definição de trajetórias de aprendizagem e no design instrucional.

Para fins de validação, presente metodologia foi apresentada para um grupo de oito doutorandos em Informática na Educação que, após utilizá-la para o desenvolvimento de um OA, respondeu a um questionário sobre a aplicabilidade da mesma. A Tabela 5-1 a seguir mostra os resultados obtidos (escores de 1 a 5 (1Discordo, 5-Concordo).

Observe que, não obstante tratar-se de um grupo de doutorandos, os participantes informaram ter pouca experiência com o projeto de Objetos de Aprendizagem Multimodais. Mesmo com pouca experiência, consideraram a 
metodologia de fácil entendimento e capaz de facilitar o projeto de Objetos de Aprendizagem eficazes e pretendem utilizá-la no futuro.

Tabela 5-1. Resultados da Pesquisa de Opinião

\begin{tabular}{|l|c|c|}
\hline Questão (1-Discordo, 5 - Concordo) & Média & $\begin{array}{c}\text { Desvio } \\
\text { Padrão }\end{array}$ \\
\hline $\begin{array}{l}\text { Tenho experiência com o Projeto de Objetos de } \\
\text { Aprendizagem Multimodais }\end{array}$ & 2,4 & 1,3 \\
\hline $\begin{array}{l}\text { O Método de Projeto de OAs Baseado em Trajetórias } \\
\text { de Aprendizagem é de fácil entendimento. }\end{array}$ & 4,3 & 0,7 \\
\hline $\begin{array}{l}\text { O uso desta metodologia facilita o projeto de Objetos } \\
\text { de Aprendizagem Eficazes. }\end{array}$ & 4,3 & 0,7 \\
\hline Pretendo utilizar esta metodologia no futuro. & 4,3 & 0,9 \\
\hline
\end{tabular}

O Quadro 5-1 apresenta algumas opiniões expressas na forma de texto livre, que reforçam os resultados apurados na Tabela 5-1.

Contribui para a organização e distribuição das tarefas no contexto de um projeto para a construção de OAs.

Facilita a criação de OAs inclusive por pessoas que tenham menos experiência na construção dos mesmos.

Possibilita aos professores serem autores de Materiais Didáticos Digitais, deixando de serem apenas consumidores.

\section{Quadro 5-1. Pesquisa de Opinião}

\subsection{Trabalhos Futuros}

A consolidação da metodologia proposta está intimamente relacionada ao software $A B C$ Autoria, cuja versão alfa encontra-se em fase final de construção. Já existem especificações para uma atualização desta metodologia, de forma a incorporar etapas de aprendizagem por descoberta, bem como diagnóstico sobre o potencial de aprendizagem significativa de um determinado OA para um determinado estudante.

\section{Referências Bibliográficas}

ABNT - ASSOCIAÇÃO BRASILEIRA DE NORMAS TECNICAS. Sistemas de gestão da qualidade - Fundamentos e vocabulário. ABNT - Associação Brasileira de Normas Tecnicas. [S.l.]. 2005. (NBR ISO 9000:2005).

AUSUBEL, D. P.; NOVAK, J. D.; HANESIAN, H. Psicologia educacional. 2. ed. Rio de Janeiro: Interamericana, 1978.

BADDELEY, A. D. Working memory. Current Biology, v. 23 , p. 136-140, 2010. BLOOM, B. S. The 2 Sigma Problem: The search for methods of group instruction as effective as one-to-one tutoring. Educational Researcher, p. 4-16, 1984.

CANTO FILHO, A. B. et al. Objetos de Aprendizagem no Apoio à Aprendizagem de Engenharia: Explorando a Motivação Extrínseca. RENOTE. Revista Novas Tecnologias na Educação, 10, 2012. 1-10.

CANTO FILHO, A. B. et al. Um Sistema para Classificação de Objetos de Aprendizagem MCS - Multimodality Classification System. Revista Novas Tecnologias na Educação, v. 12, p. pdf, 2013. ISSN ISSN 1679-1916. 
CANTO, A. B. et al. Recomendações de projeto de objetos de aprendizagem: em busca da autonomia na aprendizagem. TISE - Congreso Internacional de Informática Educativa. Santiago - Chile: [s.n.]. 2012. p. 315-323.

CANTO, A. B. et al. Classificação de Objetos de Aprendizagem Segundo o Grau de Multimodalidade. RENOTE - Revista Novas Tecnologias na Educação, Por, v. 11, p. 1-10, 2013.

CANTO, A. B. et al. Tecnologia, Descoberta e Recepção: um Estudo de Caso. XLI Congresso Brasileiro de Educação em Engenharia. Gramado - RS: [s.n.]. 2013. p. pdf.

CANTO, A. B.; ET.AL. Objetos de aprendizagem na educação em engenharia. In: AL.], V. F. D. O. (. ).. [. Desafios da educação em engenharia: formação em engenharia, internacionalização, experiências metodológicas e proposições. 1. ed. Brasília-DF: ABENGE, 2013a. Cap. VI, p. 311-359.

CANTO, A. B.; LIMA, J. V.; TAROUCO, L. M. R. Objetos de Aprendizagem Indutiva: Um Estudo de Caso. Octavas Conferencia Latinoamericana de Objetos y Tecnologías de Aprendizaje. Valdivia - Chile: [s.n.]. 2013b. p. 71-82.

FELDER, R. M.; PRINCE, M. Inductive Teaching and Learning Methods: Definitions, Comparisons, and Research Bases. Journal of Engineering Education, 2006.

KALYUGA, S.; RIKERS, R.; PAAS, F. Educational implications of expertise reversal effects in learning and performance of complex cognitive and sensorimotor skills. Educational Psychology Review, v. 24, p. 313 -337, 2012.

MAYER, R. E.. I. M. R. E. (. ). Introduction to multimedia learning. In: MAYER, R. The Cambridge Handbook of Multimedia Learning. New York: Cambridge University Press, 2005.

MAYER, R. E.; HEISER, J.; LONN, S. Cognitive Constraints on Multimedia Learning: When Presenting More Material Results in Less Understanding. Journal of Educational Psychology, 93(1), 2001. 187-198.

MORENO, R.; MAYER, R. Interactive multimodal learning environments. Educational Psychology Review, v. 19, p. 309-326, 2007. ISSN ISSN: 1573-336X.

SUNG, E.; MAYER, R. E. When graphics improve liking but not learning from online lessons. Computers in Human Behavior, v. 28, p. 1619-1625, May 2012.

SWELLER, J. Cognitive load during problem solving: Effects on learning. Cognitive Science, v. 12, p. 257-285, 1988.

SWELLER, J.; G., V. M. J. J.; C., P. F. G. W. Cognitive architecture and instructional design. Educational Psychology Review, v. 3, p. 251-296, 1998.

TAROUCO, L. M. R.; SILVA, C. C. G.; GRANDO, A. Fatores que afetam o reuso de objetos de aprendizagem. Renote - Novas Tecnologias em Educação, v. 9, p. pdf, 2011.

VAN MERRIËNBOER, J. J. G.; SWELLER, J. Cognitive load theory in health professional education: design principles and strategies. Medical Education, v. 44, p. 85-93, 2010. 